\title{
Infant timbre perception: Discrimination of spectral envelopes
}

\author{
MARSHA G. CLARKSON, RACHEL K. CLIFTON, and EVE E. PERRIS \\ University of Massachusetts, Amherst, Massachusetts
}

\begin{abstract}
Seven-month-old infants were trained to discriminate the timbre of tonal complexes that differed in their spectral envelopes. In a conditioned head-turning paradigm, infants initially learned to discriminate stimuli that had the same fundamental frequency, $200 \mathrm{~Hz}$, but different harmonic components (i.e., frequencies). After successfully completing the basic timbre discrimination, the same infants learned to discriminate less salient stimuli from which the fundamental frequency had been removed. These results suggest that young infants can analyze the spectra of tonal complexes and discriminate differences in one of the most important cues for timbre perception in adults, the spectral envelope.
\end{abstract}

Complex tones can differ in three primary attributes: loudness, pitch, and timbre. Whereas the loudness and pitch of a sound depend primarily on a single physical parameter, the total sound pressure level and the fundamental frequency, respectively, timbre is a multidimensional attribute. No single semantic scale describes qualitative differences in timbre, and no single physical parameter underlies these differences. Consequently, less empirical data exist and perceptual models are less explicit for timbre than for loudness and pitch (Plomp, 1970).

Despite its complexity, timbre provides important information for listeners' interactions with and organization of their auditory worlds. For example, the characteristic qualities of musical instruments, which render them recognizable in a variety of contexts, reflect timbre. Regardless of the note it sounds, a clarinet's quality remains constant and differs from that of a violin. Likewise, the multidimensional quality of a vowel distinguishes it from other vowels. As with musical instruments, a given vowel (e.g., /a/) spoken by various persons whose voices have different pitches retains its identifiable quality. Adults apparently utilize the same perceptual mechanisms for distinguishing the timbre of musical sounds and the quality of vowels (Plomp, 1976).

Numerous physical characteristics are employed by these perceptual mechanisms to code the timbre of sounds, but multidimensional scaling studies of adults' perceptions have begun to reduce those characteristics to a manage-

This research was supported by Grant BNS 8304419 from the National Science Foundation to R. K. Clifton and M. G. Clarkson and by Grant MH-00332 from the National Institute of Mental Health to Clifton. We thank Haskins Laboratories in New Haven, CT, for use of their facilities for stimulus construction and Alison Block, Peter McLaughlin, Adrienne Paine, and Gloria Patti for their help in data collection and analysis. Finally, we thank all the infants and parents who participated in this research. Requests for reprints should be sent to Marsha Clarkson, Department of Psychology, University of Massachusetts, Amherst, MA 01003 able number (e.g., Grey, 1977; Miller \& Carterette, 1975; Plomp, 1970). From those studies, the spectral envelope of sounds emerges as the single most important cue for timbre (de Bruijn, 1982; Preis, 1984). The spectral envelope is defined as the curve connecting the points that represent the amplitudes of stimulus components in a tonal complex. The shape of the spectral envelope can be changed in a variety of ways by manipulating the amplitudes of stimulus components and by varying the frequencies of those components. For adults, the latter manipulation produces especially salient differences in timbre. Listeners describe sounds that contain high-frequency energy as "brighter" or "sharper" (Bismark, 1974) than sounds that contain predominantly low frequencies. For example, when they sound the same note, an oboe would be termed brighter than a bassoon.

Because timbre provides an important source of organization for auditory information, the ontogenesis of our ability to perceive timbre should be determined. When is the young infant able to discriminate sounds on the basis of timbre? What acoustic information is used by the young infant in his/her discrimination? In the present experiment, we evaluated 7-month-old infants' discrimination of the timbre of tonal complexes. Little direct evidence exists for timbre discrimination during infancy, although several studies have shown that infants discriminate vowel quality. Newborn infants can discriminate isolated vowels (Clarkson \& Berg, 1983), and by 1-4 months of age, infants reliably discriminate both naturally spoken (Trehub, 1973) and synthetically produced vowels (Kuhl \& Miller, 1982; Swoboda, Morse, \& Leavitt, 1976). Any attempt to specify infants' acoustic abilities on the basis of these vowel-discrimination studies, however, is difficult. First, infants' discrimination of speech sounds is inextricably tied to the development of language, and performance depends on both acoustic processing and experience with language. Furthermore, the complex spectral information that determines vowels makes the acoustic basis of infants' performance unclear. 
One way to delineate the spectral cues that infants can use is to present simple, nonspeech stimuli, such as tonal complexes, and then selectively add spectral and temporal information. Such a tack isolates the acoustic processing used in timbre perception.

Indirect evidence of timbre perception emerges from an earlier study of infants' extraction of pitch from tonal complexes (Clarkson \& Clifton, 1985). In that study, 7 month-old infants learned to discriminate the pitch of tonal complexes and then categorized several "instruments" on the basis of their pitch. When the multiple instruments were introduced, however, both the number of false alarms and the number of spontaneous turns during the background increased. Such increases would occur only if the infants discriminated the timbre of the varying exemplars. In another study of infants' pitch perception, Bundy, Colombo, and Singer (1982) varied the timbre of tonal complexes but did not directly test for timbre discrimination.

In the present experiment, we used an adaptation of a conditioned head-turning paradigm (see Kuhl, 1985) to test 7-month-old infants' ability to discriminate the timbre of sounds with different spectral envelopes. All temporal parameters of the stimulus components were the same across stimuli so that only differences in the spectral envelope could provide a basis for discrimination. Our earlier pitch-perception study employed both stimuli that contained the fundamental frequency and signals that lacked it. The fundamental frequency is a unique component in tonal complexes and does not always behave in the same manner as other components. In fact, removing the fundamental frequency from a tonal complex reduces the salience of both the pitch and timbre of the sound more than does removing any other single component. Hence, we included missing-fundamental sounds in this study to determine whether the result would be poorer discrimination of timbre.

\section{METHOD}

\section{Subjects}

Infants were recruited from published birth announcements via a letter and follow-up telephone call. All subjects met criteria for an uncomplicated term birth, good health on the day of testing, and $n$ history of chronic ear infections or suspicion of hearing loss. Tu nty-two (12 female, 10 male) 7-8-month-old infants ( $M=32.39$ wee. ', range $=29.57-35.43$ weeks) constituted the sample. The data $t_{t}-8$ other infants were excluded because of equipment malfunction (1), schedule conflicts for return visits (3), and failure to produce three consecutive unassisted responses within the first 48 trials of shaping (4).

\section{Stimuli}

All tonal complexes were synthesized at Haskins Laboratories in New Haven, Connecticut, as described in Clarkson and Clifton (1985). The 500-msec-duration stimuli had 50-msec rise/fall times and occurred once per second. Due to equipment limitations, stimuli and intertrial intervals, which ranged from 6 to $11 \mathrm{sec}$, were taperecorded. One channel of these tapes contained stimuli, and a second channel contained $250-\mathrm{msec}, 1000-\mathrm{Hz}$ tones that signaled trial onset to the other equipment. Stimuli were presented at a sound pressure level of $45 \mathrm{dBA}$, and the ambient noise level was $28 \mathrm{dBA}$, as measured at the approximate position of the infant's head with a Brüel \& Kjaer Model 2204 sound level meter and a 1-in. 4132 condenser microphone. No measureable changes in overall SPL occurred when the stimuli changed.

The six $200-\mathrm{Hz}$ tonal complexes were identical to those employed in a previous study of pitch perception (Clarkson \& Clifton, 1985). The complexes differed in the number and the frequencies of the harmonic components they contained and, therefore, differed in their spectral envelopes. All components were of equal amplinude and were combined in cosine phase. Figure 1 depicts the frequency components for each of the tonal complexes used in various stages of the experiment. 'The discrimination stimuli that were used to shape responding and establish discrimination contained the fundamental frequency plus six consecutive harmonics: (1) harmonics 5 to 10 , frequencies ranging from 1000 to $2000 \mathrm{~Hz}$, for the background envelope; (2) harmonics 7 to 12 , frequencies ranging from 1400 to $2400 \mathrm{~Hz}$, for the high envelope; and (3) harmonics 3 to 8 , frequencies ranging from 600 to $1600 \mathrm{~Hz}$, for the low envelope. The number of harmonics was limited to six because the tonality of tonal complexes decreases as higher harmonic numbers are included (Ritsma \& Hoeckstra, 1974). Three missing-fundamental stimuli contained only six consecutive harmonics (see Figure 1). The frequency ranges differed for the discrimination and missing-fundamental stimuli so that infants could not rely on memory for specific frequencies in the first stage as a basis for responding in the subsequent one.

\section{Apparatus}

The infant sat on a parent's lap at a small table in a sound-deadened chamber, and an assistant, who entertained the infant with attrac-

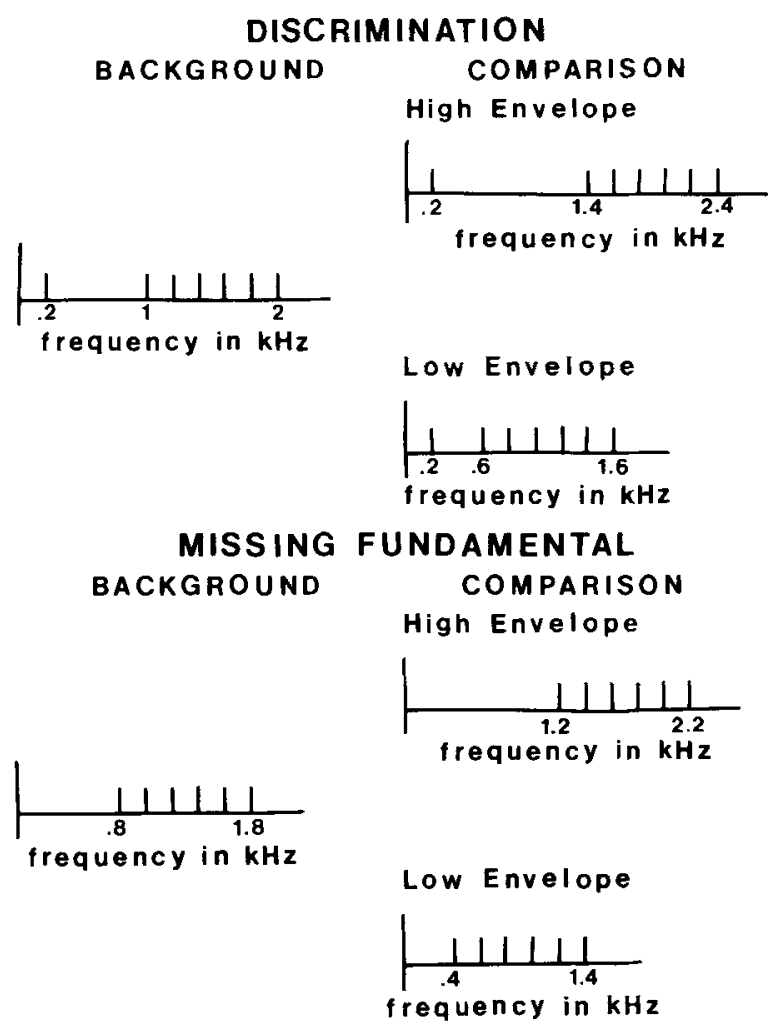

Figure 1. Stimulus components for the background and comparison stimuli used in the discrimination and missing-fundamental stages of the experiment. The background stimulus contained an intermediate spectral envelope for both experimental conditions, whereas the comparison stimulus consisted of the high spectral envelope for one condition and the low spectral envelope for the other. All stimuli had a pitch of $200 \mathrm{~Hz}$. 
tive toys, sat opposite the infant. An experimenter in an adjoining room controlled the equipment and observed the infant on a video monitor. Stimulus tapes were played from a Teac X7-R tape recorder and fed through a Pioneer SX-450 amplifier to an AR-7 loudspeaker that was mounted on the wall approximately $1.5 \mathrm{~m}$ to the infant's right or left (position was counterbalanced across subjects) and at a $60^{\circ}$ angle to him or her. Two visual reinforcers were placed in front of and below the loudspeaker. The reinforcers consisted of commercially available toy animals that were housed in dark, smoked-Plexiglas boxes and could not be seen until lights inside the boxes were illuminated.

A Northstar Horizon computer and interfaced BRS Series 200 logic detected trial onset and controlled the infant's progress through the experiment. Throughout each trial, the computer activated one small light in the test chamber out of the infant's view and another small light in the adjoining room. The logic detected the experimenter's and assistant's judgments of head turning, and the computer recorded those votes, activated the reinforcer at the appropriate times, and determined when the infant had met criterion for each stage.

\section{Procedure}

General Procedure. The current procedure was similar to that used by others to test auditory sensitivity and discrimination (see Kuhl, 1985). During the experiment, a background stimulus (see Figure 1) was played repeatedly with 6-sec trials interspersed among those stimuli. On change trials, the background stimulus was replaced by one of the comparison stimuli (either the high-envelope stimulus or the low-envelope one), but on control trials, the background stimulus remained. While infants learned the task in shaping, intertrial intervals ranged from 7 to $11 \mathrm{sec}$, which allowed the assistant time to direct the infant's attention away from the reinforcer when it went off and back to his/her toys before the next trial began. By the end of shaping, the infants typically looked back to the assistant after the reinforcer stopped, so intertrial intervals were shortened to 6 to $10 \mathrm{sec}$ for the remainder of the experiment.

During each trial, the assistant and the experimenter independently pressed a footpedal or button if the infant turned his/her head. They did not vote if the infant was already looking toward the reinforcers at the start of a trial. When both the assistant and the experimenter voted that the infant had turned toward the sound, the infant was credited with a turn. If the turn occurred at any time during a change trial, the reinforcer was activated for $5 \mathrm{sec}$. Turns during control trials or intertrial intervals were not reinforced. If neither the assistant nor the experimenter or if only one of them voted that a turn had occurred, the infant was not credited with a turn. We scored a correct response if the infant: (1) turned on a change trial, or (2) did not turn on a control trial.

Experimental stages. Infants progressed through three successive stages: shaping, discrimination, and missing fundamental. They were randomly assigned to two timbre-discrimination conditions, for which the background spectral envelope was contrasted with either the higher envelope or the lower one.

During shaping, several procedures were employed to help the infant learn the task. To provide a high level of reinforcement, we presented only change trials in this stage. The assistant cued the baby when the timbre change occurred and, across trials, provided successively fewer cues until the infant turned without assistance. When the infant had made unassisted turns on three consecutive trials, testing proceeded to the discrimination stage. Each infant received at least 10 and a maximum of 48 trials to complete shaping.

In the discrimination stage, half the trials were change trials as described above and half were control trials on which no stimulus change occurred. Trial order was randomized, with the restriction that no more than two consecutive trials of a given type occur. Although this restriction assured the possibility of a high reinforcement rate, it could also have resulted in an artificially depressed false-alarm rate. Whenever two consecutive trials were reinforced, the assistant could know that a control trial was coming and alter her vote to fit the trial type. Such a voting bias did not emerge in the data, however; the false-alarm rates for control trials following two reinforced trials (.337) and for all other control trials (.340) were virtually equivalent. Infants met criterion in this and the subsequent stages when they responded correctly on 9 of 10 consecutive trials, including both change and control trials. In this stage, and for the remainder of the experiment, the parent and assistant listened to music over headphones so that they could not cue the infant that a stimulus change had occurred. Since the assistant knew only when a trial occurred, not the type of trial, neither her criterion for judging head turns nor her way of entertaining the infant could systematically differ with trial type. The experimenter could, however, hear the stimuli. Thus, any problems with the computer, stimulus tapes, or other equipment could be quickly detected and remedied. Since the votes of both the experimenter and the assistant were automatically recorded, any systematic bias on the part of the experimenter could be observed. The votes of the experimenter and the assistant agreed on $96.9 \%$ of the trials in the discrimination stage and on $97.8 \%$ of the trials in the missingfundamental one that followed.

The missing-fundamental stage differed from the preceding one in that stimuli now lacked the fundamental frequency. The relative spectral envelopes of the sounds, however, remained the same as in the discrimination stage. For example, infants in the low-envelope condition continued to hear comparison stimuli with a lower spectral envelope than that of the background stimuli. The introduction of the missing-fundamental stimuli, with their novel spectral envelopes, confused some of the infants. If infants did not turn on four consecutive change trials or turned on two of three consecutive control trials, they were "primed" with the presentation of discrimination stimuli. Since they had already successfully discriminated these stimuli, the infants were expected to quickly reorient to the task. They received up to $\mathbf{1 0}$ trials to reach a criterion of correct responding on three of four consecutive trials. When this occurred, testing returned to the missing-fundamental stage. If infants did not reach criterion in priming within 10 trials, we assumed that fatigue may have interfered with their performance, and we ended their sessions and scheduled return visits. On return visits, infants received a series of shaping trials with the discrimination stimuli, after which testing resumed with the missing-fundamental stimuli.

Infants received up to four series of trials in the missingfundamental stage, with priming stimuli presented between each series. If infants reached criterion in the priming procedure three times but still did not respond correctly on 9 of 10 consecutive trials for the missing-fundamental stage in the fourth series of trials, we concluded that they had failed the missing-fundamental task. All infants completed testing in one to three visits.

\section{RESULTS}

The present data were analyzed in two different ways. At a group level, discrimination was tested by comparing the number of turns on change and control trials. For those infants (16) who were tested in both the discrimination and missing fundmental stages, an analysis of variance on the number of turns included experimental condition (high envelope, low envelope) as a between-subject factor and stage (discrimination, missing fundamental) and trial type (change, control) as within-subject factors. Separate analyses included the data for all subjects who had received stimuli in each stage, regardless of whether they had met criterion in that stage or had been tested in a subsequent stage. Thus, 22 infants contributed to the analysis for the discrimination stage and 16 contributed 
to the missing-fundamental one. These group analyses provide the primary evidence of timbre discrimination. In addition, the trials-to-criterion data permit us to look at each individual infant's performance to determine how many infants contributed a reliable discrimination performance to the group data. Since the statistical reliability of criterion performance depends upon both the number of trials received and the level of chance responding, we assessed the probability that each infant met criterion by chance. These analyses included data only from infants who actually met criterion in each stage (i.e., 16 infants for the discrimination stage and 11 infants for the missingfundamental one).

\section{Change vs. Control Responding}

Overall, infants tested in both stages turned more often on change than on control trials [trial type $F(1,14)=$ $148.87, p<.0001]$, but the trial type effect did not significantly interact with either experimental condition or stage. Inclusion of data for all subjects showed that infants turned significantly more often on change $(M=$ 11.14) than on control $(M=6.59)$ trials in the discrimination stage $[t(21)=6.72, p<.001]$. Likewise, for the missing-fundamental stimuli, more turns occurred on change $(M=10.56)$ than on control $(M=6.38)$ trials $[t(15)=8.21, p<.001]$. At the start of each stage, infants produced false alarms on approximately 2 of 5 control trials, but these unreinforced turns decreased as headturn behavior came under stimulus control. The mean number of control trials on which infants turned for successive blocks of 10 trials is plotted for each stage in
Figure 2 (dashed line). As the number of false alarms decreased across trials, the number of turns on change trials increased (solid lines in Figure 2).

\section{Trials to Criterion}

On the average, infants completed the shaping stage within 17.35 trials (range $=10$ to 35 ). An analysis of variance (ANOVA) that included experimental condition (high envelope, low envelope) as a between-subject factor revealed no significant difference in the number of shaping trials. Sixteen of the 22 infants met criterion in the discrimination stage within 10 to 61 trials $(M=28.1)$, while 11 of those infants (68.75\%) passed the missingfundamental stage. On the average, they required 36.63 trials (range $=10$ to 88) to do so. No significant differences due to condition or stage emerged from an ANOVA on the number of trials to criterion.

Although the majority of infants tested in the discrimination and missing-fundamental stages met criterion, some of them may have done so on the basis of chance turning. To assess this possibility statistically, we estimated the likelihood that each infant met criterion in each stage by chance and then determined the probability that the infant proceeded through the entire experiment by chance. Since all turns were toward the loudspeaker and equal numbers of change and control trials occurred, we took the rate of turning on control trials as the level of chance responding for each infant in each stage. Priming stimuli were used merely to determine whether infants would continue to perform the task, so data for those trials did not contribute to the criterion analyses. Using the probabil-

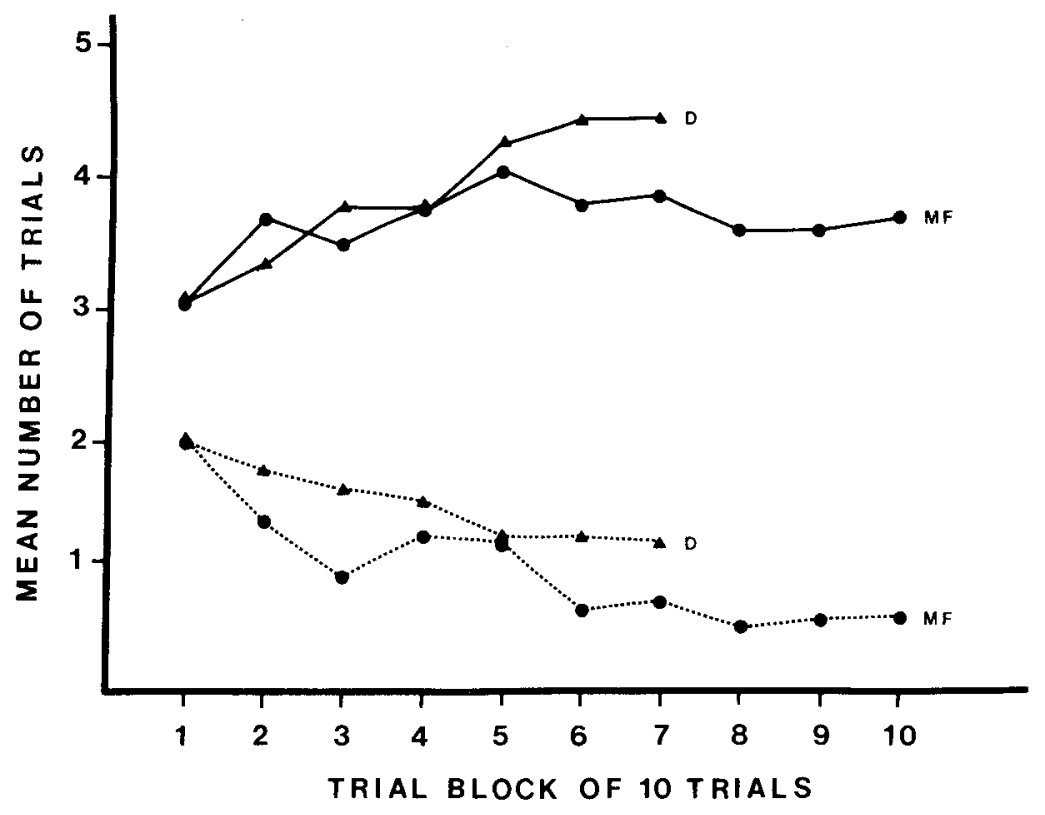

Figure 2. Mean number of trials on which infants turned for change trials (solid lines) and control trials (dashed lines). Data are plotted for each test stage in blocks of 10 trials containing 5 change and 5 control trials. The curves labeled $D$ represent the discrimination stage $(N=22)$, and those labeled MF depict the missing-fundamental one $(N=16)$. 
ity formulas presented in Bogartz (1965), we estimated, on the basis of his/her level of chance responding, the likelihood that each infant met criterion by chance in the number of trials received. For each infant, the probability of completing the entire experiment on the basis of chance was computed by multiplying the probabilities for meeting criterion in each stage. In the discrimination and missing-fundamental stages, the probabilities of reaching criterion by chance ranged from 0.0 to 0.25 and 0.0 to 0.42 , respectively. The likelihoods of completing the entire experiment on the basis of chance responding ranged from 0.0 to 0.04 . Summing the probabilities across subjects, both for each stage and for the entire experiment, estimates the number of infants in our sample who could have met criterion with only random responding. Less than 2 infants (sum of probabilities $=1.6$ ) could have completed the discrimination stage by chance, whereas less than 1 (sum of probabilities $=0.21$ ) could have done so for the missing-fundamental stage. For the entire experiment, less than 1 infant (sum of probabilities $=0.05$ ) could have successfully finished by chance.

\section{DISCUSSION}

The present experiment provides a first step toward understanding infants' perception of timbre and of the acoustic cues employed therein. As a group, 7-month-olds successfully discriminated sounds that differed in their spectral envelopes. The infants performed equally well whether the range of frequencies in the comparison sounds was higher or lower than that of the background. When the fundamental frequency was removed from the sounds, the group evidenced discrimination of the changes in spectral envelope with these less salient stimuli. Nonetheless, fewer individual infants met the performance criterion for the missing-fundamental stimuli than for the discrimination ones. These individual data suggest that the infants may have had more difficulty with the missingfundamental stimuli, but because no significant differences in group performance emerged for the two stages, we have no statistical evidence that the stimuli in the two stages differed in discriminability. The variations in individual performance were also not large enough to prevent the entire group from showing a reliable difference between change and control trials. Although some infants did not evidence reliable discrimination, as a group, 7-month-old infants discriminated the timbre differences in tonal complexes.

The stimuli for the present experiment were kept as simple as possible so that we could isolate the acoustic basis of the infants' discrimination. For these stimuli, the harmonic content alone provided sufficient acoustic information for infants to discriminate the sounds. Whether infants utilized the entire range of the spectral envelope or directed their attention to isolated frequency regions of those envelopes is unclear. Infants might have successfully discriminated the sounds by monitoring the energy in specific regions, for example below the fifth harmonic. Increased energy in that region would indicate the timbre change in the low-envelope condition, while decreased energy would cue the change in the high-envelope one. If infants employed this strategy, they would not need to code the entire shape or frequency range of the spectral envelope. Although we do not know what strategy adults employ in timbre discrimination, the ability to selectively monitor high- or low-frequency regions may partially account for the importance of the harmonic content dimension for adults' timbre perception. Our infants' performance reveals a similar sensitivity to the harmonic content (and therefore spectral envelope) as one basis on which to discriminate timbre.

In addition to the spectral envelope, two other acoustic properties related to the harmonic content of the sounds might account for the infants' performance. First, the background and comparison stimuli share four common components with two components unique to each stimulus. Infants might have isolated and based their performance on these unique components, but this possibility seems unlikely. Although highly trained adults can isolate up to the fifth harmonic (equivalent to $1000 \mathrm{~Hz}$ here) from tonal complexes, they have difficulty extracting higher harmonics because of limitations in the auditory system's capacity to resolve frequencies (Plomp, 1964). In a masking paradigm, Olsho (1985) demonstrated that 6-month-old infants' frequency resolution does not differ from that of adults. Consequently, we would expect one component $(1000 \mathrm{~Hz})$ for the high-envelope group and two components $(600$ and $800 \mathrm{~Hz})$ for the low-envelope group to have been available to our infants. If the infants isolated and used these unique frequencies, then the additional unique information in the low-envelope stimuli should have made them easier to discriminate than the high-envelope ones. Since no differences in performance emerged for the two spectral envelope groups, isolated frequencies are unlikely to have provided the basis for the infants' discrimination.

Another potential basis for our infants' discrimination arises from differences in the subjective loudness of the stimuli. Adults perceive loudness differences for stimuli that contain the same number of equal amplitude components when, as in our stimuli, those components span different numbers of critical bands. Calculations of loudness for our stimuli revealed that no loudness difference should exist between the background and high-envelope discrimination stimuli, but that a small (about equivalent to $3 \mathrm{~dB}$ ) difference should exist between the background and low-envelope discrimination stimuli as well as both pairs of missing-fundamental stimuli. Although infants' coding of subjective loudness has not been studied, it is reasonable to postulate that their coding is similar to that of adults. In this case, if infants used loudness differences as a basis for discrimination, a very specific pattern of results should have emerged. First, in the discrimination stage, infants in the low-envelope group should have performed better than infants in the high-envelope one. Indeed, we would not have expected infants in the latter group to have discriminated at all, because there was no loudness difference. Second, infants in the high-envelope 
group should have discriminated the missing-fundamental stimuli but not the discrimination ones, inasmuch as only the missing-fundamental stimuli provided a loudness cue. Neither of these results emerged, so a loudness explanation of the infants' discrimination seems unlikely. Rather, infants must have extracted and discriminated the spectral envelopes of the sounds.

The application of multidimensional scaling techniques to adults' perception of tonal complexes reveals that the spectral envelope is the most important determinant of timbre (Bismark, 1974; Plomp, 1970; Plomp \& Steeneken, 1969). Although the harmonic content is the dominant cue (de Bruijn, 1978), many other factors, including the bandwidth of the signal, the balance of energy in the lower harmonics, and the existence of the upper harmonics, also influence the spectral envelope. Manipulating these more subtle cues will clarify whether infants actually code the shape of the spectral envelope or can attend only to isolated regions of the envelope. With the more complex sounds of musical instruments, onset transients and other temporal cues provide additional information about timbre (Grey, 1977). A complete understanding of infants' timbre discrimination will require manipulating both the spectral cues in tonal complexes and the temporal information in more complex musical sounds.

\section{REFERENCES}

Bismark, G. vON (1974). Timbre of steady sounds: A factorial investigation of its verbal attributes. Acustica, 30, 146-159.

BogarTZ, R. S. (1965). The criterion method: Some preliminary analyses and remarks. Psychological Bulletin, 64, 1-14.

Bundy, R. S., Colombo, J., \& Singer, J. (1982). Pitch perception in young infants. Developmental Psychology, 18, 10-14.

Clarkson, M. G., \& Berg, W. K. (1983). Cardiac orienting and vowel discrimination in newborns: Crucial stimulus parameters. Child Development, 54, 162-171.

Clarkson, M. G., \& Clifton, R. K. (1985). Infant pitch perception: Evidence for responding to pitch categories and the missing fundamental. Journal of the Acoustical Society of America, 77, 1521-1528.

DE BruUn, A. (1978). Timbre-classification of complex tones. Acustica, 40, 108-114.
GREY, J. M. (1977). Multidimensional perceptual scaling of musical timbres. Joumal of the Acoustical Society of America, 61, 1270-1277. KuHL, P. K. (1985). Methods in the study of infant speech perception. In G. Gottlieb \& N. A. Krasnegor (Eds.), Measurement of audition and vision in the first year of postnatal life: A methodological overview (pp. 223-251). Norwood, NJ: Ablex.

KUHL, P. K., MilleR, J. D. (1982). Discrimination of auditory target dimensions in the presence or absence of variation in a second dimension by infants. Perception \& Psychophysics, 31, 279-292.

Miller, J. R., \& Carterette, E. C. (1975). Perceptual space for musical structures. Journal of the Acoustical Society of America, 58, 711-720.

Olsho, L. W. (1985). Infant auditory perception: Tonal masking. Infant Behavior \& Development, 8, 371-384.

Plomp, R. (1964). The ear as a frequency analyzer. Journal of the Acoustical Society of America, 36, 1628-1636.

Plomp, R. (1970). Timbre as a multidimensional attribute of complex tones. In R. Plomp \& G. F. Smoorenburg (Eds.), Frequency analysis and periodicity detection in hearing (pp. 397-411). Leiden: Sijthoff.

Plomp, R. (1976). Aspects of tone sensation: A psychophysical study. London: Academic Press.

Plomp, R., \& Steeneken, H. J. M. (1969). Effect of phase on the timbre of complex tones. Journal of the Acoustical Society of America, 46, 409-421.

PREIS, A. (1984). An attempt to describe the parameter determining the timbre of steady-state harmonic complex tones. Acustica, 55, 1-13.

Rutsma, R. J., \& Hoeckstra, A. (1974). Frequency selectivity and the tonal residue. In E. Zwicker \& E. Terhardt (Eds.), Facts and models in hearing (pp. 156-163). New York: Springer.

Sworoda, P. J., Morse, P. A., \& LeavitT, L. A. (1976). Continuous vowel discrimination in normal and at risk infants. Child Development, 47, 459-465.

TREHUB, S. E. (1973). Infants' sensitivity to vowel and tonal contrasts. Developmental Psychology, 9, 91-96.

\section{NOTE}

1. The spectra of representative exemplars of each stimulus complex were examined by analyzing with a Tektronix $5 \mathrm{~L} 4 \mathrm{~N}$ spectrum analyzer the output of a Brüel \& Kjaer 4125 1-in. condenser microphone placed at the approximate position of the infant's head. These analyses indicated that the tonal complexes contained the appropriate components and that the amplitudes of the components were equal to within $0.5 \mathrm{mV}$. Thus, retaping the stimuli did not create significant distortions, and the loudspeakers did not recreate the fundamental frequency.

(Manuscript received December 1, 1986; revision accepted for publication June 22, 1987.) 